\title{
The Campi Flegrei Deep Drilling Project
}

\author{
by Giuseppe De Natale, Claudia Troise, and Marco Sacchi
}

doi:10.2204/iodp.sd.4.15.2007

\section{Introduction}

Large collapse calderas represent an extremely explosive type of volcanism with potential for global impact. Although very rare, such super-eruptions represent a serious threat to mankind. However, moderate to small eruptions from such calderas represent a major risk as well, particularly in urban areas. One example is the 1994 eruption of the Rabaul caldera which destroyed the town of Rabaul (Papua New Guinea). This event demonstrated that eruptions are possible with only minor and very short-term precursory signals, while long episodes of unrest involving uplifts on the order of up to 1 meter and intense seismicity may occur without immediate volcanic activity.

Campi Flegrei in Southern Italy is a typical example of a collapsed caldera whose surface is partially $(60 \%)$ submerged (Fig. 1). It has the highest recorded ground movements not immediately followed by eruptions. First colonized by Greeks and home to the largest Mediterranean military fleet during the Roman times (Baia, on the west side of caldera), this area has the longest historical record of ground movements associated with volcanic activity, as revealed by marine incrustations and molluscs on the Roman and medieval buildings (Fig. 2 and Troise et al., 2006). The secular deformation of this area is subsidence, at a rate of about $1.5-1.7 \mathrm{~cm} \mathrm{yr}^{-1}$, with some periods of large and faster uplift (Fig. 2A). Historically, three such uplift periods are evident; the first one occurred from ca. 650 to $730 \mathrm{AD}$ and the second from 1441 to 1538 , while the third one is still in progress following the latest eruptions since 1969 (Fig. 2B). This recent period is characterized by episodes of very fast deformation rates, followed by periods of minor subsidence (Fig. 2C). The total uplift from 1969 to 1984 was $3.5 \mathrm{~m}$, while the uplift rate in the period 1983-1984 exceeded $1 \mathrm{~m} \mathrm{yr}^{-1}$. A relatively fast subsidence was recorded after 1985 until the end of 2004; since late 2005 a new uplift episode is in progress,

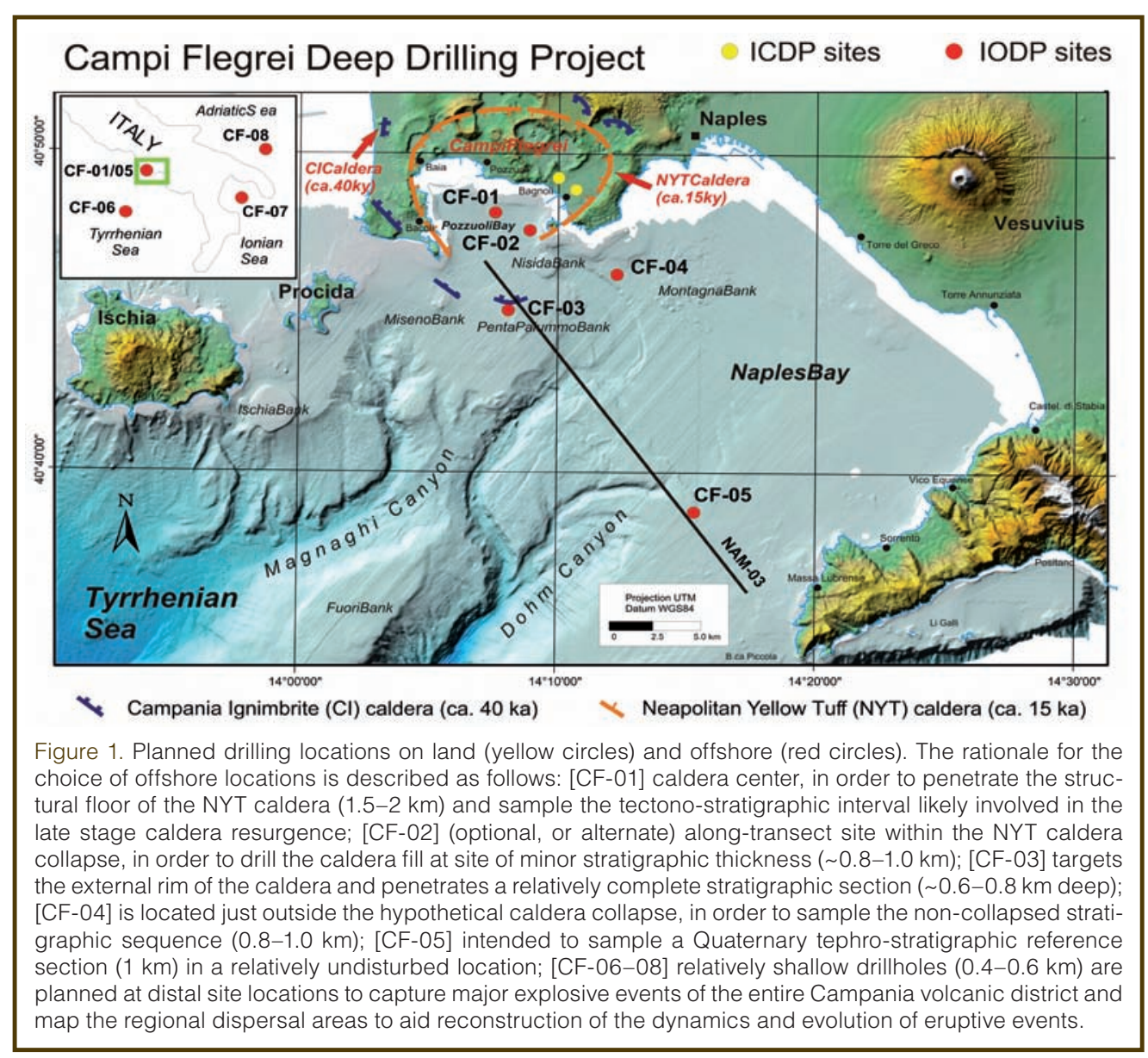
currently reaching $4 \mathrm{~cm}$, half of which in the last two months of the record (Fig. 2C).

Thelargestcaldera-forming eruption occurred about 39,000 years ago, when grey tuff covered the whole Campania region with large flow deposits. Associated fine ashes can be traced as far away as Moscow (Russia). The most recent caldera-forming eruption (15,000 years ago) produced a yellow tuff deposited over the larger Naples region. More than sixty smaller hydromagmatically eruptions modified this area up to present time. With a history of massive volcanic eruptions and ongoing deformation, this densely urban area including the city of Naples is exposed to a very high volcanic risk. 


\section{Planning Workshop}

A workshop on the 'Campi Flegrei Deep Drilling Project' was held on 13-15 November 2006 in Naples, Italy, in a location within the Campi Flegrei caldera and close to the main candidate site for on-land drilling-the Bagnoli area (Fig. 1). The workshop was among the first European efforts to assess volcanic hazard within the context of the Integrated Ocean Drilling Program (IODP) and the International Continental Scientific Drilling Program (ICDP). The participants presented evidence that the setting and features of the partially submerged Campi Flegrei Caldera provides an ideal natural laboratory for future joint ICDP-IODP research. About seventy scientists from nine countries representing multidisciplinary fields participated in the meeting. A plenary session was held on the first day, followed by groups for onland and off-shore drilling working separately during the second and third days to define science targets and appropriate tools for an integrated amphibious drilling program. Important on-going ICDP and IODP drilling projects in areas with objectives and technologies of interest to the Campi Flegrei project were presented during joint sessions. These included keynotes on the regional volcanic evolution and results of previous geothermal drilling activities. The different working groups ranked main scientific objectives and elaborated the best strategies for drilling and related research in the respective fields. In addition, the development of new drilling proposals for IODP and the nurturing of existing proposals were discussed.

\section{Main Conclusion}

A key conclusion from the workshop was that the combined on-land and off-shore drilling is required to address the following key scientific objectives: 1) to understand the link between shallow fluids and magmatic systems generating unrest and feeding extremely explosive eruptions, 2) to better assess the high volcanic risk in the area and to develop best strategies for risk mitigation in Campi Flegrei and in similar environments, and 3) to develop new technologies of environmental monitoring and the exploitation of geothermal energy including industrial spin-offs.

The workshop participants pointed out that in order to accomplish the main targets, a strict integration of several research fields is needed, including geology, geophysics, seismology, petrology, geochemistry, geochronology, geothermal investigations, numerical and analogue modelling, physics of the matter, and engineering. The multidisciplinary project structure will require the combination of several work packages tied to and supporting the central tool of deep drilling and coring. For on-land drilling, the most
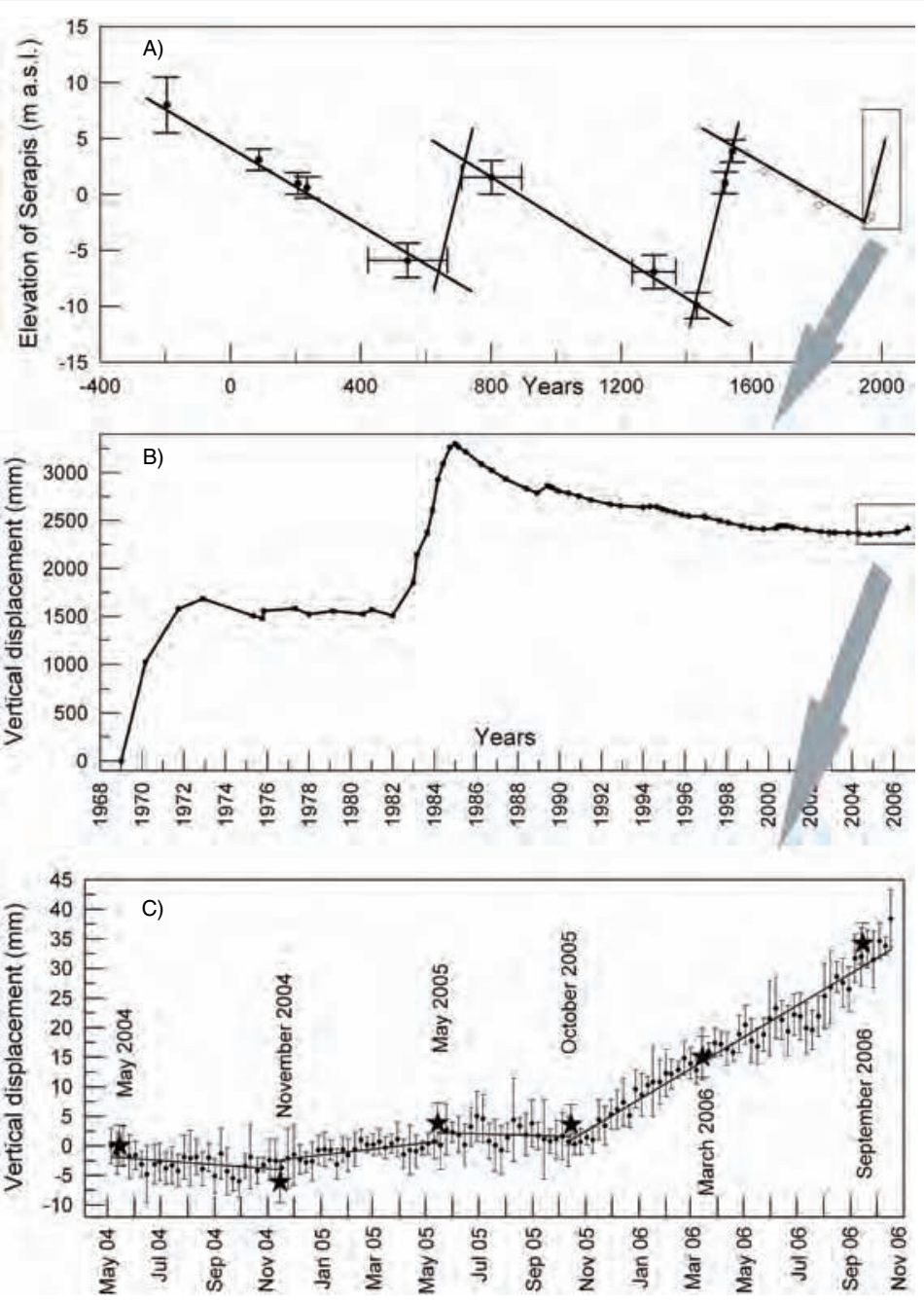

Figure 2. Ground deformation at Campi Flegrei as a function of time, at three progressive enlargements. [A] Schematic vertical movement history at Macellum in Pozzuoli, known as Serapis Temple. Black circles and white circles represent data points from various sources (see De Natale et al., 2006 and references therein). [B] Vertical ground displacements as recorded at Pozzuoli Harbor by leveling data during 1969-2006. [C] Detail of vertical displacement from May 2004 to October 2006 as recorded from CGPS (dots) and precision leveling at bench mark in Pozzuoli harbor (stars). Errors on CGPS and leveling data (10) are also shown (Redrawn after Troise et al., 2006).

reliable site has been identified in the area of Bagnoli (Fig.1). This site is large enough to host two drill sites-one close to the caldera rim, and the other closer to the caldera center. The first drill site on land close to the caldera rim allows for further addressing two important scientific objectives with a relatively shallow hole $(<2 \mathrm{~km})$ : 1) understanding of the rheology of an aseismic layer which seems to represent a strongly fractured and water-saturated medium with a sharp transition down towards a seismically active, gas-saturated medium, and 2) direct observation of mechanical and fluiddynamical properties across the caldera borders which have been interpreted as sites of focused stress and strain with dominant ductile (aseismic) behavior (De Natale et al., 2006).

The second and deeper drill hole on land closer to the caldera center will additionally target the deeper geothermal system and heat flow (including the critical temperature 
point, estimated at $2.5-3 \mathrm{~km}$ depth) to measure the purely conductive gradient and to extrapolate magmatic temperatures (target depth $=4-4.5 \mathrm{~km}$ ).

In order to study in detail the properties of the rocks and the geothermal system for the whole depth extension at the caldera center (position CF-1), this drilling should be complemented by an off-shore site into the caldera center down to the critical point at this position (estimated depth $\sim 2 \mathrm{~km}$ ). A second (maximum 1-km-deep) off-shore site is planned just outside the caldera border (southeast of the Posillipo hill, in the Gulf of Naples, position CF-4 in Fig. 1). The goal will be to compare the different structure and rheology across the caldera border and to sample a complete section of the older volcanic successions. Additional off-shore sites are planned to complete the reconstruction of Campi Flegrei and larger Campania volcanism by deep drilling, as specified in the caption of Figure 1.

\section{Acknowledgements}

The Campi Flegrei Workshop was mainly sponsored by ICDP and the ESF within the framework of Magellan workshops. The meeting was organized and partially supported by INGV and IAMC-CNR with the contribution of
AMRA-Campania, University of Naples Federico II, and INOA-CNR.

\section{References}

De Natale, G., Troise, C., Pingue, F., Mastrolorenzo, G., Pappalardo, L., Battaglia, M., and Boschi, E., 2006. The Campi Flegrei Caldera: unrest mechanisms and hazards. In Troise, C., De Natale, G., and Kilburn, C.R.J., (Eds.), Mechanisms of Activity and Unrest at Large Calderas. Special Publication 269:159-171, London (Geological Society Library).

Troise, C., De Natale, G., Pingue, F., Obrizzo, F., De Martino, P., Tammaro, U. and Boschi, E., 2007. Renewed ground uplift at Campi Flegrei caldera (Italy): new insight on magmatic processes and forecast. Geophys. Res. Lett., 34, L03301, doi:10.1029/2006GL028545.

\section{Authors}

Giuseppe De Natale and Claudia Troise, INGV - Via Diocleziano 328, 80124 Naples, Italy, e-mail: denatale@ov. ingv.it.

Marco Sacchi, IAMC-CNR - Calata Porta di Massa, Porto di Napoli, 80133 Naples, Italy. 